\title{
The origin of the radio emission from $\beta$ Lyrae
}

\author{
G. Umana ${ }^{1}$, F. Leone ${ }^{2}$, and C. Trigilio ${ }^{1}$ \\ ${ }^{1}$ Istituto di Radioastronomia del C.N.R., Stazione VLBI di Noto, C.P. 161 Noto, Italy \\ 2 INAF, Osservatorio Astrofisico di Catania, via S. Sofia 78, 95123 Catania, Italy \\ Received 5 October 2001 / Accepted 15 May 2002
}

\begin{abstract}
In this paper we present new observational evidence that supports the presence of an extra source of continuum emission in the binary system $\beta$ Lyrae. New VLA and IRAM observations, together with published data from the literature and ISO archive data, allow us to build the Spectral Energy Distribution of the binary between $5 \times 10^{9} \mathrm{~Hz}$ and $5 \times 10^{15} \mathrm{~Hz}$. The radio-millimeter part of the spectrum is consistent with free-free emission from a symbiotic-like wind associated with the primary component and ionized by the radiation field of the hidden companion. Furthermore, we also consider the possibility that the observed radio flux originates from collimated radio structures associated with the mass gaining component and its disk (Conical thermal jets). An extrapolation of this emission to the far-IR part of the spectrum indicates that in both cases the free-free contribution at these frequencies cannot explain the observations and that the observed infrared excess flux is due principally to the secondary component and its associated disk.
\end{abstract}

Key words. stars: binaries: eclipsing - stars: individual: $\beta$ Lyrae - radio continuum: stars

\section{Introduction}

Even if the eclipsing binary $\beta$ Lyrae is one of the most studied stellar systems, its enigmatic spectroscopic and photometric behaviour is not fully understood. The current view is a non-degenerate, semi-detached interacting binary system in the phase of large-scale mass transfer between components. The mass losing component is a B6-B8p II, while the unseen mass gaining component is probably a B0V star, embedded in an optically thick accretion disk (Hubeny \& Plavec 1991). The presence of a large plasma cloud, surrounding both components, has been inferred from optical and UV emission lines (Batten \& Sahade 1973; Hack et al. 1975) as well as from the analysis of UV light curves (Kondo et al. 1994). Recently the presence of jet-like structures, probably related to the accretion disk, has been shown by the studies of Harmanec et al. (1996) and confirmed by Hoffman et al. (1998). $\beta$ Lyrae is also a well known radio source. First detected, in the early seventies, by Wade \& Hjellming (1972) at 2.7 and $8.1 \mathrm{GHz}$, it was then monitored, at two frequencies, by Gibson (1975). The source always exhibited a thermal-like spectrum but Wright \& Barlow (1975) pointed out that the observed slope of the radio spectrum $(\alpha=0.96)$ was intermediate between the slope expected for a simple $\mathrm{H}_{\text {II }}$ region and from a stellar wind, implying that the physics underlying the radio emission of $\beta$ Lyrae is more complicated than assumed in either of these models.

Recent high resolution MERLIN observations of $\beta$ Lyrae at $4.9 \mathrm{GHz}$ (Umana et al. 2000) have revealed an extended radio nebula around the system, whose brightness temperature

Send offprint requests to: G. Umana,

e-mail: umana@ira.noto.cnr.it $\left(1.1 \times 10^{4} \mathrm{~K}\right)$ confirms the thermal origin of the radio emission. Such a nebula can be re-conducted to a massive wind associated with the cooler primary ionized by the hotter secondary if the two-winds model by Mazzali et al. (1992) is adopted.

To assess a clear picture of the radio properties of $\beta$ Lyrae, an analysis of its spectral energy distribution (SED) from radio to infrared appears to be necessary. In this paper we present multi-frequency VLA and IRAM observations that will be combined with data from the literature in order to investigate the origin of the radio emission of $\beta$ Lyrae.

In the following, assuming the most common notation, we will indicate as primary the mass losing component, as it is the most luminous in the optical region.

\section{Observations and results}

\subsection{The VLA data}

The observations were carried out using the $\mathrm{VLA}^{1}$ on May 5, 1996, from 07:58 to 12:46 UT. We observed $\beta$ Lyrae at five frequencies, namely 4.8 ( $C$-band), 8.4 ( $X$-band), 14.9 ( $U$-band) and 22.0 ( $K$-band) and 43.0 ( $Q$-band) $\mathrm{GHz}$, using two independent $50 \mathrm{MHz}$ bands. The observations were performed in compact (D) configuration, splitting the array in two different subarrays: the first one included all the antennas (13) equipped with $Q$-band receivers; the other subarray was used sequentially at each remaining frequency. This ensures the necessary

\footnotetext{
1 The Very Large Array is a facility of the National Radio Astronomy Observatory which is operated by Associated Universities, Inc. under cooperative agreement with the National Science Foundation.
} 
Table 1. VLA observations.

\begin{tabular}{cccc}
\hline \hline Band [GHz] & Flux density [mJy] & rms [mJy] & U.T. \\
\hline 4.8 & 4.27 & 0.06 & $10: 40$ \\
8.4 & 6.50 & 0.05 & $11: 00$ \\
14.9 & 9.60 & 0.20 & $10: 00$ \\
22 & 12.70 & 0.30 & $11: 20$ \\
43 & 13.90 & 0.50 & $10: 00$ \\
\hline
\end{tabular}

sensitivity for detection of weak sources. The adopted antenna configuration provides a typical beam size of $\sim 12^{\prime \prime}, \sim 7.5^{\prime \prime}$, $\sim 4^{\prime \prime}, \sim 2.5^{\prime \prime}$ and $\sim 3^{\prime \prime}$ at 4.8, 8.4, 14.9, 22 and $43 \mathrm{GHz}$ respectively.

A typical observing cycle consisted of 15-20 min integration time, preceeded and followed by a 2-min observation of the phase calibrator. This basic sequence was repeated at least 3 times in order to improve the signal to noise ratio.

For $Q$-band observations a slightly different observing strategy was followed. Frequent checking for pointing was performed since systematic errors may be a significant part of the primary beam at $43 \mathrm{GHz}$. Moreover, in order to minimise atmospheric effects on the phases, a much shorter observing cycle has been adopted. As phase calibrator, 1925+211 was chosen for the $Q$-band, while for the other frequencies we used $1850+284$. The flux density scale was determined by observing $3 \mathrm{C} 286$. The $43 \mathrm{GHz}$ flux of $1925+211$ was determined relative to $3 \mathrm{C} 286$ by using only those scans obtained at the same elevation as for 3C286. This ensures a careful amplitude calibration since at high frequencies there is a strong dependence on elevation of the antenna aperture efficiency and of the atmospheric opacity.

The data processing was performed using the standard programs of the NRAO Astronomical Image Processing System (AIPS). To achieve the highest possible signal to noise ratio, the mapping process was performed by using the natural weighting and the dirty map was CLEANed down as close as possible to the theoretical noise. At each frequency, the source position and the flux density determination were obtained by fitting a gaussian brightness distribution (JMFIT). To estimate the noise level in the maps we analyzed an area on the map, with dimensions of $\sim 15-20 \theta_{\text {syn }}$, away from the phase center. Its consistency with the expected theoretical noise was always acertained.

We detected $\beta$ Lyrae at all the 5 frequencies as a compact, unresolved source. Our results are summarized in Table 1, where the radio flux density, with its associated rms and the time of the observation are reported for each different frequency. In spite of the fact that $\beta$ Lyrae was one of the first stars to be detected in the early seventies, the measurements here reported are the first detections of the system at high frequencies.

\subsection{The IRAM data}

Observations of the millimeter continuum of $\beta$ Lyrae were obtained in 1996, between 15 and 16 of May, with the IRAM ${ }^{2}$

\footnotetext{
${ }^{2}$ IRAM is supported by INSU/CNRS (France), MPG (Germany) and IGN (Spain).
}

Table 2. IRAM-PdB observations.

\begin{tabular}{cccc}
\hline \hline Band [GHz] & Flux density [mJy] & rms [mJy] & U.T. \\
\hline 85 & 26.8 & 0.7 & $5: 13$ \\
115 & 27.0 & 4.0 & $4: 54$ \\
215 & 36.9 & 4.5 & $5: 13$ \\
240 & 38.0 & 3.0 & $4: 54$ \\
\hline
\end{tabular}

Plateau de Bure (PdB) interferometer (Guilloteau et al. 1992). These observations were carried out in a compact configuration (D), providing a typical beam size of $5^{\prime \prime} \times 8^{\prime \prime}$ at $1.3 \mathrm{~mm}$. Since each of the four operating antenna was equipped with a SIS dual-frequency receivers at 1.3 and $3 \mathrm{~mm}$, the observations were performed simultaneously at $85 \mathrm{GHz}$ and $215 \mathrm{GHz}$ (on May 15) and at $115 \mathrm{GHz}$ and $240 \mathrm{GHz}$ (on May 16). The observations were made in double-sideband (DSB) mode, with each sideband of $500 \mathrm{MHz}$ separated by $3 \mathrm{GHz}$.

A typical observation consisted of $20 \mathrm{~min}$ on source scan, plus several calibration measurements to assure an accurate calibration of the interferometer, bandpass and phase calibration and a high pointing accuracy. This sequence was repeated several times, for a total of about four hours source integration time, in order to improve the signal to noise ratio. Phase calibration was performed by using $2013+370$ while the flux scale was fixed by using daily observations of the primary calibrators MWC349 and 3C454.3. The IRAM data were reduced and mapped following the standard procedures using the software developed by the Observatoire de Grenoble and IRAM (Grenoble Image and Line Data Analysis Software)

We detected $\beta$ Lyrae at all the 4 frequencies. The results are summarized in Table 2, where the millimetric flux density, with its associated rms, is reported for each different frequency.

\section{Spectral energy distribution}

$\beta$ Lyrae has been observed by the ISO satellite and we retrieved three scientifically validated spectra obtained with the Short Wavelengths Spectrograph (SWS) from November 1997 to March 1998. The spectra were successively analyzed using the ISAP package. This software was specifically written for the reduction and scientific analysis of ISO SWS and LWS (Long Wavelengths Spectrograph) Auto Analysis Results (AARs), which is the final output of the standard ISO pipeline processing.

As a final step, we estimate the continuum flux in 6 different channels by choosing areas free of spectral lines and evaluating the average flux by applying a box-car filter of $2 \mu$ width. This analysis was performed only in the spectrum with the best signal to noise ratio.

$\beta$ Lyrae is also positionally associated with the IRAS source $18482+3318$ and, as pointed out by Friedmann et al. (1996), has a spectral distribution in the IRAS bands typical of circumstellar material.

Figure 1 shows the Spectral Energy Distribution (SED) of $\beta$ Lyrae built using our reported observations, referenced measurements from the literature as well as unpublished archive data. 


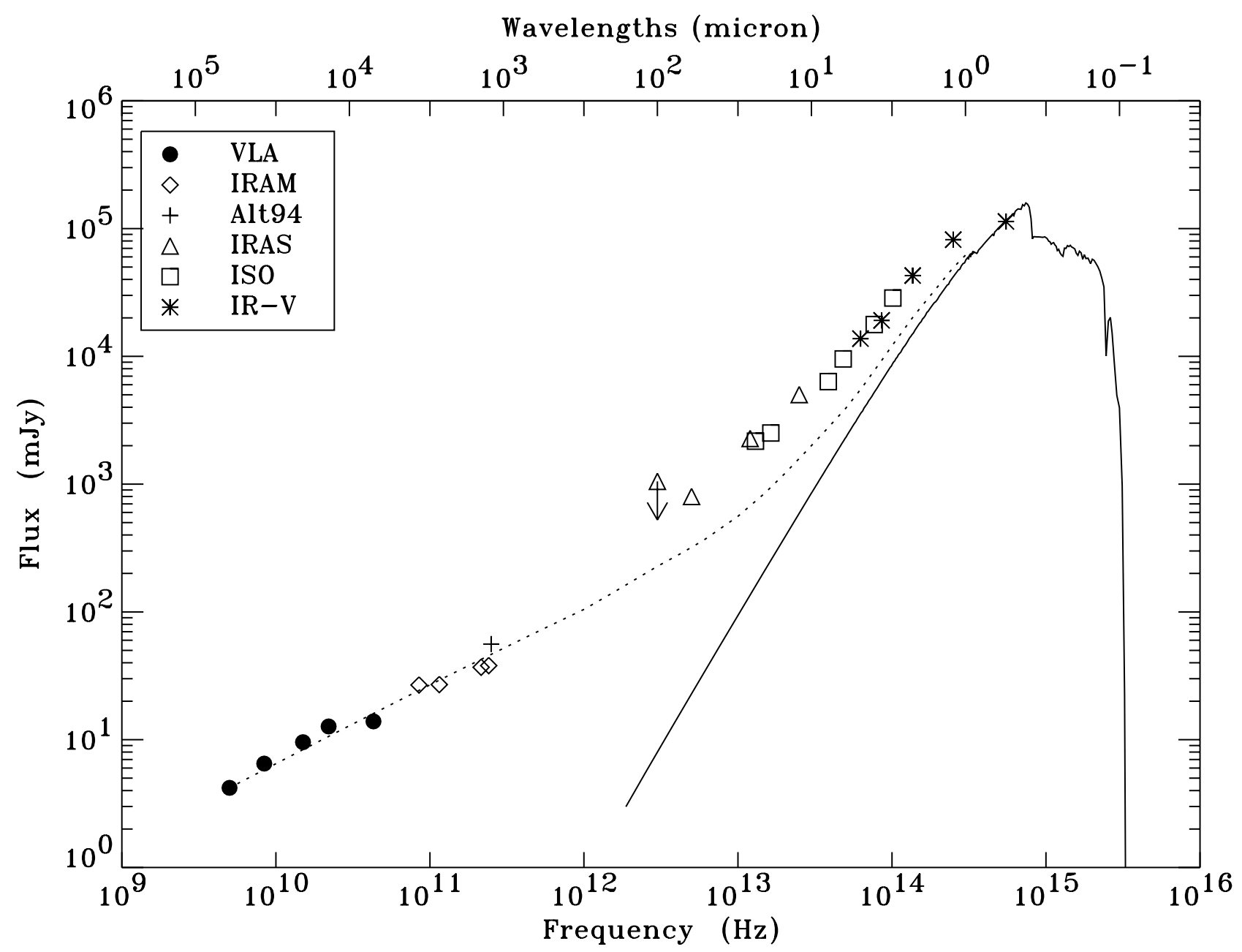

Fig. 1. The SED of $\beta$ Lyrae. Dots, diamonds and the crosses represent VLA and IRAM data from this paper plus a datum from Altenhoff et al. (1994). Triangles and squares indicate IRAS and ISO data, while asterisks are VJKLM magnitudes from Viotti et al. (1978), $V$ magnitude has been corrected as in the text. Data points have been corrected for interstellar extinction following Mathis (1990) and assuming a $E_{B-V}=0.065$. The dotted line is the contribution at high frequencies of the free-free plus bound-free emission from the stellar wind. The stellar contribution is represented by an ATLAS9 (Kurucz 1993) model, with fluxes normalized to the flux in the $V$ band (solid line).

$\beta$ Lyrae is reported in the literature as a periodically variable source, whose ephemeris are (Harmanec \& Scholz 1993):

$$
T=\mathrm{JD} 2408247.966+12.91378 E+3.87196 \times 10^{-6} E^{2}
$$

and questions may arise regarding whether it is possible to combine data obtained at different orbital phases.

In order to take in account the variability with the orbital phase in building the SED, we should consider only observations carried out at orbital phases close to those of our VLA observations, corresponding to orbital phase $\Phi \sim 0.151 \pm 0.016$.

For the infrared and visible, measurements obtained at orbital phases between 0.14 and 0.16 and corrected for an interstellar extinction of $E_{B-V}=0.065$ (Abt et al. 1962) have been used.

For the ISO and IRAS spectral regions, information on possible variation with orbital phase is not available, but it is very probable that at longer wavelengths the contribution of the circumsystem material would be important and the resultant continuum will be less affected by orbital variation. Given the amplitude of light curves decreasing from the visible to the IR, proceeding in a conservative way, if we use the eclipse depths reported by Zeilik et al. (1982) for the infrared as dispersion associated with each IRAS and ISO datum, we end up with an errorbar smaller than the symbols used in the plot of Fig. 1.

Finally, we can built up the radio-mm spectrum quite confidentily, even if the source has been reported variable at these frequencies and the data were not obtained simultaneously, since a VLA radio flux monitoring of $\beta$ Lyrae did not reveal any variations at least on a time-scale of a month or so (Umana et al. 2002). Moreover our millimetric measurements are in agreement with those obtained at $250 \mathrm{GHz}$ by Altenhoff et al. (1994) more than 15 years ago.

Same secular changes of the radio flux density of $\beta$ Lyrae are present, as reported by several authors. However, in the following analysis of the spectral distribution we allow the flux density at $5 \mathrm{GHz}$ to change between 2.9 and $4.3 \mathrm{mJy}$, minimum and maximum measured $5 \mathrm{GHz}$ flux density, retaining the spectral index determined in the present work. This leads to the conclusion that taking secular changes into account does not affect our main results. 


\section{Discussion}

The radio spectrum of $\beta$ Lyrae can be well represented by a single power-law. The best fit of the cm data is $S_{v} \propto v^{(0.62 \pm 0.02)}$, consistent with an optically thick thermal source and very close to the canonical spectral index value as expected from a stellar wind.

This result is quite different from what was claimed by Wright \& Barlow (1975) who derived a spectral slope of 0.96 from observations of $\beta$ Lyrae in the early seventies carried out at 2.3 and $8.4 \mathrm{GHz}$. We attribute the difference between this and our result to the large experimental errors associated with the old measurements.

Jameson \& King (1978) made the first attempt to model the radio emission of $\beta$ Lyrae by assuming a homogeneous, spherically symmetric $\mathrm{H}$ II region. This model, which assumes an emitting region embracing the entire system, foresees a black body spectral index and a turnover frequency at $84 \mathrm{GHz}$, which is not evident from our observations.

\subsection{Stellar wind/s}

The UV spectrum of $\beta$ Lyrae has been known to be dominated by anomalous continuum and very strong emission lines (Hack et al. 1975). The observed emission features, which show unusually strong P-Cyg profiles, can be divided in two groups. The first group contains lines of highly ionized species, typical of hot-star winds, with a broad underlying emission. The other group includes lines of species at lower ionization stages (Aydin et al. 1988).

A two-wind radiation-driven model has been proposed by Mazzali et al. (1992) to explain these two different kinds of mass loss features. The different characteristics suggest two distinct regions where they form, the first associated with the wind of the B0 V gainer, the second in the wind of the B6-8 II loser.

The possibility that the radio emission could be related to the interactions between the two stellar winds is ruled out by the fact that the Mazzali et al. (1992) model outlines a system where only one of the winds is dominant. This would imply that the effects of possible wind interactions are negligible (Stevens 1995). Moreover, the observed radio spectrum is consistent with thermal emission rather than non-thermal as observed in most colliding wind radio sources (Williams 1996).

Recently Umana et al. (2000) resolved an extended radio nebula, embracing the entire binary system, by using the MERLIN interferometer at $4.9 \mathrm{GHz}$. In this paper the authors suggested that it is possible to interpret the observed nebula, in the framework of the Mazzali et al. (1992) hypothesis, as a result of the the stellar wind associated with the BII component ionized by the hotter companion. Such a phenomenon is observed in Symbiotic systems where the associated radio nebula is the material ejected by one of the components which is ionized by the secondary star.

The radio properties of symbiotics are well summarized in a series of papers (see for example, Seaquist \& Taylor 1990). In particular, the observed radio spectra have been modelled in terms of a binary model, where the morphology of the radio emitting region is a function of the physical characteristics of the system such as binary separation $(a)$, Lyman continuum luminosity of the hot component $\left(L_{\mathrm{uv}}\right)$, mass loss rate $(\dot{M})$ and velocity $(v)$ of the dominant wind (Taylor \& Seaquist 1984 TS).

If the wind is completely ionized, the ionized region shows the same radio properties as a spherically symmetric ionized wind. Thus, in the framework of the TS model, a spectral index close to the canonical 0.6, as we observed in $\beta$ Lyrae, would imply that at least a large fraction of the wind is ionized. A large ionization region can be written in the TS terminology as $q_{\mathrm{o}} a<<a$, where $q_{\mathrm{o}}$ is the distance, measured in terms of the binary separation $a$, from the center of the mass-losing star and the boundary of the ionized region. Using this assumption, from Eq. (8) of TS, we can relate the turnover frequency to the physical parameters of the system by the equation:

$v_{\mathrm{t}}^{2.1}>>8 \times 10^{66}\left(\frac{T_{\mathrm{e}}^{-1.35}}{a^{3}}\right)\left(\frac{\dot{M}}{v}\right)^{2}$

where $a$ is in cm, $\dot{M}$ in $M_{\odot} \mathrm{yr}^{-1}, v$ in $\mathrm{km} \mathrm{s}^{-1}$.

Assuming $a=58.5 R_{\odot}$ (Bisikalo et al. 2000), the $\dot{M}$ and $v$ values of the dominant wind (BII component, see Table 3 ) and $T_{\mathrm{e}}$ value obtained by MERLIN observations (Umana et al. 2000), we derive a turnover frequency higher than $760 \mathrm{GHz}$.

The observed spectrum, which is optically thick up to $\sim 300 \mathrm{GHz}$, is thus consistent with the symbiotic interpretation.

The far IR part of the spectrum is more difficult to interpret mostly because at these frequencies the contribution of the photosperic emission becomes important.

The primary star in $\beta$ Lyrae is spectroscopically clearly visible and has been classified as a B8-6 II (Balachandran et al. 1986). On the contrary very little is still known about the nature of the secondary nother than the fact that it should be embedded in an accretion disk. However, despite of the numerous efforts (Wilson 1974; Linnell \& Hubeny 1996; Linnell et al. 1998; Linnell 2000), there is no standard model of an accretion disk able to reproduce synthetic light curves that fit the observed ones from IR to UV.

Therefore, we can compute the expected far-IR flux only for the primary component by assuming, between $1.5 \times 10^{16}$ and $1.5 \times 10^{12} \mathrm{~Hz}$ a Kurucz (1993) model with $T_{\text {eff }}=13000 \mathrm{~K}$, $\log (g)=3$, as appropriate for the B8-6 II component.

Since the observed continuum is the sum of the primary and secondary continuum, to normalize the atmospheric model we correct the observed $V$ magnitude, as observed by Viotti et al. (1978), by a factor 1.10. This has been calculated by assuming a flux ratio between the two components at maximum light of 3 (Harmanec 1990) and a further decrement of about $12 \%$ of the primary's flux due to the fact that the observations took place at orbital phase $\Phi=0.15$.

The radio emission from a stellar wind comes from regions quite far from the star, where the wind velocity has reached its terminal velocity $\left(v_{\infty}\right)$. Because of the dependence of thermal opacity on wavelengths $\left(\kappa_{\lambda} \propto \lambda^{2}\right)$, emission at higher frequencies would come from regions much closer to the stellar photosphere, where some acceleration takes place. Thus to evaluate the contribution of such a stellar wind to the far-IR part of the 


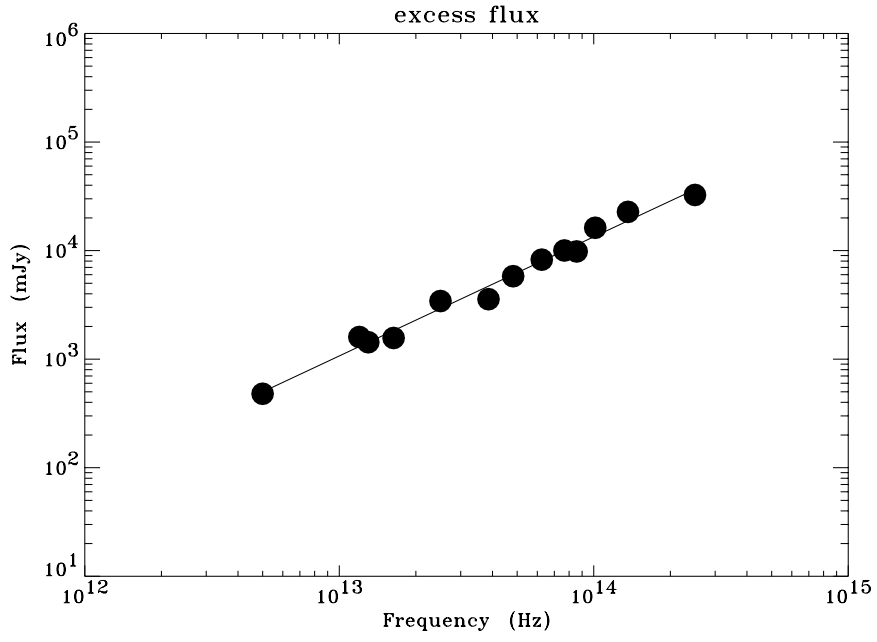

Fig. 2. The excess flux of $\beta$ Lyrae. The line is the fit of the contribution at high frequencies of star+disk system.

spectrum, the effects of the velocity law of the velocity of the mass-flow must be taken into account.

Lamers \& Waters (1984) have computed the energy distribution for a grid of expanding, isothermal stellar wind models. In their work they considered all the possible ways the extended envelope can affect the energy distribution of the central star, modelling quite accurately the far-IR part of the spectrum.

In adapting the Lamers \& Waters (1984) model to the two winds scenario of $\beta$ Lyrae we will assume that the secondary component plays only the role of providing the radiation field necessary to ionize the wind associated with the primary. Following them, we thus derive the emission due to the star+wind system, by assuming as physical parameters of the stellar wind those of the dominant wind, that of the B8-6 II component, as derived by Umana et al. (2000). For the wind, we assume that the velocity is a function of the radial distance, in units of $R_{*}$ :

$\frac{v(x)}{v_{\infty}}=0.01+0.99\left(1-\frac{1}{x}\right)$

as suggested by spectroscopic UV observations (Castor \& Lamers 1979).

The obtained energy distribution, normalized at $5 \mathrm{GHz}$, is shown in Fig. 1 (dotted line), where it is evident that the observed flux starts to deviate from the expected $\propto v^{2}$ photospheric behavior at $\sim 5 \times 10^{14} \mathrm{~Hz}$ and there is an extra far-IR excess flux that the presence of a stellar wind cannot explain.

This excess, extracted and plotted in Fig. 2, can be fitted by a power law:

$S_{v} \propto v^{1.10 \pm 0.04}$

in agreement, surprising, with the results from Viotti et al. (1978), which, however, were based only on JKLM observations reported in the plot. As already pointed out by Viotti and co-authors, this result is consistent with a secondary hidden by an accretion disk, whose dimension increases with wavelength.
Table 3. Binary and Nebula characteristics.

\begin{tabular}{lcc}
\hline \hline Binary $^{\mathrm{a}}$ & Gainer & Loser \\
\hline$T_{\text {eff }}[\mathrm{K}]$ & 32000 & 13300 \\
$\dot{M}\left[M_{\odot} \mathrm{yr}^{-1}\right]$ & $4.68 \times 10^{-8}$ & $7.16 \times 10^{-7}$ \\
$v_{\infty}\left[\mathrm{km} \mathrm{s}^{-1}\right]$ & 1470 & 388 \\
Radio Nebula $^{\mathrm{b}}$ & & \\
\hline$T_{\mathrm{e}}[\mathrm{K}]$ & $11000 \pm 700$ & \\
$S i z e(F W H M)[\mathrm{mas}]$ & $(145 \pm 12) \times(100 \pm 8)$ & \\
\hline a) Mazzali et al. $(1992)$. & \\
b) Umana et al. (2000). & \\
\end{tabular}

\subsection{Thermal jets}

The two-wind model of Mazzali has been recently criticized by Harmanec (2002), who pointed out that there is no evidence of wind associated with the secondary component, at least from emission or P Cygni lines as observed in the ultraviolet. Moreover, the elongated radio nebula, as observed by MERLIN, appears to be aligned with the reported optical jets, suggesting the possibility that the radio emission is due to thermal radio jets emanating from the accretion region around the hidden secondary.

Several authors have investigated the effects on radio flux, spectral index and mass-loss derivation due to deviations from sphericity in a extended stellar envelopes (Schmid-Burkg 1982; Reynolds 1986). While collimated thermal jets can produce radio spectra significantly different from that of a canonical wind, there are no spectral clues that can help in discriminating between a spherically symmetric wind or biconical constant velocity flows (wider thermal jets). Therefore, we have a piece of observational evidence that if the origin of radio emission is a thermal jet it must be not collimated.

We can derive the jet opening angle $\left(\theta_{0}\right)$ from the ratio of the minor $\left(\theta_{\min }\right)$ and major axis $\left(\theta_{\max }\right)$ of the radio nebula as derived from MERLIN measurements (Table 3).

$\theta_{0}=2 \operatorname{tg}^{-1} \frac{\theta_{\min }}{\theta_{\max }} \sim 69^{\circ}$

To determine the mass-loss of a thermal jet with opening angle $\theta_{0}$, the formula, derived in the hypothesis of symmetry in the wind, has to be corrected by a factor of $\eta=0.2 \theta_{0}(\sin i)^{-1 / 4}$ (Eq. (21) of Reynolds 1986), where $i$ is the angle of inclination of the jet as seen by the observer $\left(i \sim 85^{\circ}-88^{\circ}\right)$ and $\theta_{0}$ is expressed in radians.

Therefore, we may derive the mass-loss from the relation:

$M_{\text {sphe }}^{\cdot}=6.7 \times 10^{-4} v_{\infty} S_{6 \mathrm{~cm}}^{3 / 4} D_{\mathrm{kpc}}^{3 / 2}\left(v \times g_{\mathrm{ff}}\right)^{-0.5} \quad M_{\odot} \mathrm{yr}^{-1}$

where full ionization and cosmic abundances have been assumed, $v_{\infty}$ is the terminal velocity of the wind, $S_{6 \mathrm{~cm}}$ the observed flux density at $6 \mathrm{~cm}$ in $\mathrm{mJy}, D_{\mathrm{kpc}}$ the distance of the system, in kpc. $g_{\mathrm{ff}}$ represents the free-free Gaunt factor that, following Leitherer \& Robert (1991), can be approximated with:

$g_{\mathrm{ff}}=9.77\left(1+0.13 \log \frac{T^{3 / 2}}{v}\right)$

where $T$, in Kelvin, is the wind temperature. 


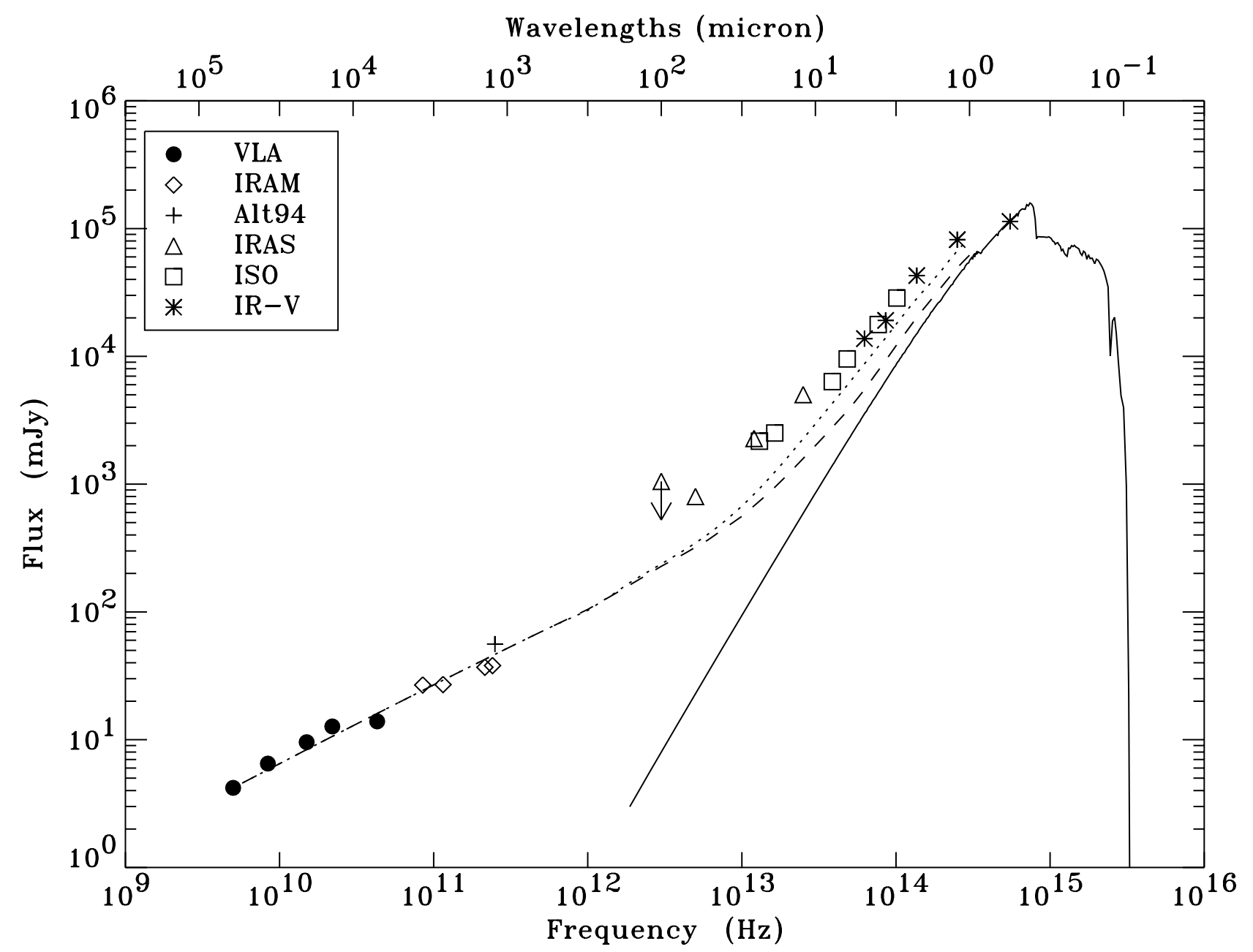

Fig. 3. Same as Fig. 1. The dotted line is the contribution at high frequencies of the free-free plus bound-free emission from a biconical outflow plus the secondary contribution represented by an ATLAS9 (Kurucz 1993) model (continuos line), with fluxes normalized to the flux in the $V$ band (solid line). For comparison the fit in the case of symbiotic wind is also shown (dashed line).

By assuming a typical stellar wind velocity ofa hot B star for the secondary component $\left(v \sim 1500 \mathrm{~km} \mathrm{~s}^{-1}\right)$ and constant temperature equal to the brightness temperature, derived by radio observations $\left(T=T_{\mathrm{B}}\right.$ ), we obtain a mass loss rate of

$M_{\text {sphe }}^{\cdot}=2.0 \times 10^{-6} \quad M_{\odot} \mathrm{yr}^{-1}$

which, corrected for thermal jets yields a mass-loss of

$\dot{M}_{\text {jet }}=\eta M_{\text {sphe }}^{\cdot}=5.2 \times 10^{-7} \quad M_{\odot} \mathrm{yr}^{-1}$.

A wide thermal jet will emit a radio flux whose frequency dependence is indistinguishable from that emerging from a spherically symmetric stellar wind. Therfore, we can apply the model of Lamers \& Waters (1984), introducted in the previous section, to derive the emission due to the star+jets system where, this time, the physical parameter of the stellar wind associated with the gainer (see Table 3) and mass-loss corrected for thermal jets have been assumed.

The obtained energy distribution, normalized at $5 \mathrm{GHz}$, is showed in Fig. 3 (dotted-line), where the contribution of the primary (as indicated by the KURUCZ model) has been also taken into account. For a better comparison, the model, obtained in the case of symbiotic winds (see Sect. 4.1) is shown in the figure as a dashed line. The model stops at $v \sim 10^{14} \mathrm{~Hz}$ as this is the highest frequency of the Lamers \& Waters (1984) tabulation.

As already noted for the symbiotic wind hypothesis, there is an extra far-IR excess flux that a thermal jet, associated with the secondary, is not able to explain. The extracted excess flux is well fitted by a power-law, with a spectral index $\alpha=0.91 \pm 0.06$, still consistent with the contribution of an accretion disk, whose density decreases with radius.

\section{Conclusions}

$\beta$ Lyrae is one of the best studied stellar systems: still no reliable model of the system, able to reproduce the huge amount of observational data, is available.

Very recently, a new generation of models, that take into consideration the details of the physics of the accretions disk, have been presented (Linnell et al. 1998; Bisikalo et al. 2000). Even if they provide a good model for the light curves in the near and far-UV and in the visible, they still do not predict the shape of the infrared light curve, and, in particular, they do not reproduce the secondary minimum that becomes deeper 
than the primary minimum at $\lambda \geq 4.8 \mu \mathrm{m}$. This leads to the conclusion that another component, besides stars plus accretion disk, must contribute to the observed flux, and Linnell (2000) proposes that the extra source of continuum radiation may be Thomson scattering of radiation from the gainer.

In this paper we have presented new observational evidence that support the presence of an extra-component in the already quite complex binary system. This extra component, which is the origin of the observed thermal radio flux, can be due to an extended stellar wind ionized by the strong ultraviolet flux of the secondary component or related to collimated structures associated with the gainer (conical thermal jets).

We further evaluated the contribution of this component in the far-IR to extract the spectral energy distribution of the stars plus accretion disk system. Our results, in both hypotheses, show a power-law distribution up to IRAS frequencies indicating an accretion disk with a non-uniform density distribution and whose size varies with wavelength. To quantify this, the collection of new, good quality infrared light curves would be highly valuable.

The stellar wind or thermal jets, as appearing from the obtained spectrum and its modelling, is optically thick up to IRAS-ISO frequencies. Still, at those frequencies we measure a significant excess that we attribute to the disk since it is in agreement with the near-IR data. We may ask what kind of effect the radio nebula may have on the observability of the inner disk. In both the cases, the size of the radio source depends on frequency as $R_{\text {radio }} \propto v^{-0.7}$. Thus, scaling the size measured at $4.8 \mathrm{GHz}$ with MERLIN (see Table 3) we obtain that the radio nebula reaches dimensions comparable to those of the entire binary system at frequencies of the order of $10^{13} \mathrm{~Hz}$, close to the IRAS spectral region.

This provides important constraints to the radio source associated with the binary system, whose morphology should allow one to observe also the disk. The other possibility, that the disk is surrounding the nebula, can be discarded, since a clear eclipse is observed in the infrared.

Finally, we would like to point out that even in the case of stellar wind, the fact that the radiation field necessary to ionize the wind is provided by the secondary component, which is embedded in the thick accretion disk (Linnell 2000), suggests that the ionization would take place mainly in the polar regions. Thus, in both the cases, it is quite probable that the nebula has a bipolar morphology that the MERLIN observations (Umana et al. 2000) were not able to resolve. This bipolar morphology, which should have a wide opening angle as suggested by the observed spectral index (Rodriguez et al. 1990), is compatible with the possibility of observing the inner accretion disk.

Acknowledgements. We acknowledge the IRAM staff from Plateau de Bure for carrying out the observations and for help provided during the data reduction. This article used archive observations with ISO, an ESA project with instruments funded by ESA Member States (especially the PI countries: France, Germany, The Netherlands and the UK) and with the participation of ISAS and NASA. The ISO Spectral Analysis Package (ISAP) is a joint development by the LWS and SWS Instrument Teams and Data Centers. Contributing institutes are CESR, IAS, IPAC, MPE, RAL and SRON. We wish to thank the referee Dr. Petr Harmanec as his suggestions greatly helped us in improving the paper.

\section{References}

Abt, H. A., Jeffers, H. M., Gibson, J., \& Sandage, A. R. 1962, ApJ, 135,429

Altenhoff, W. J., Thum, C., \& Wendker, H. J. 1994, A\&A, 281, 161

Aydin, C., Brandi, E., Engin, S., et al. 1988, A\&A, 193, 202

Balachandran, S., Lambert, D. L., Tomkin, J., \& Partharasarathy, M. 1986, MNRAS, 219, 479

Batten, A. H., \& Sahade, J. 1973, PASP, 85, 599

Bisikalo, D. V., Harmanec, P., Boyarchuk, A. A., Kuznetsov, O. A., \& Hadrava, P. 2000, A\&A, 353, 1009

Castor, J. I., \& Lamers, H. J. G. L. M. 1979, ApJS, 39, 481

Dobias, J. J., \& Plavec, M. J. 1985, AJ, 90, 773

Friedmann, C., Guerler, J., \& Loewe, M. 1996, A\&AS, 117, 205

Gibson, D. M. 1975, Ph.D. Thesis, University of Virginia

Guilloteau, S., Delannoy, J., Downes, D., et al. 1992, A\&A, 262, 624

Hack, M., Hutchings, J. B., Kondo, Y., et al. 1975, ApJ, 198, 453

Harmanec, P. 2002, AN, 323, 87

Harmanec, P. 1990, A\&A, 237, 91

Harmanec, P., Morand, F., Bonneau, D., et al. 1996, A\&A, 312, 879

Harmanec, P., \& Scholz, G. 1993, A\&A, 279, 131

Hoffman, J. L., Nordsieck, K. H., \& Fox, G. K. 1998, AJ, 115, 1576

Hubeny, I., \& Plavec, M. J. 1991, AJ, 102, 1156

Jameson, R. F., \& King, A. R. 1978, A\&A, 63, 285

Kondo, Y., McCluskey, G. E., Silvis, J. M. S., et al. 1994, ApJ, 421, 787

Kurucz, R. L. 1993, in A new opacity-sampling model atmosphere program for arbitrary abundances, ed. M. M. Dworetsky, F. Castelli, \& R. Faraggiana, Peculiar versus normal phenomena in A-type and related stars, IAU Col. 138, ASP Conf. Ser., 44, 87

Lamers, H. J. G. L. M., \& Waters, L. B. F. M. 1984, A\&A, 136, 37

Leitherer, C., \& Robert, C. 1991, ApJ, 377, 629

Linnell, A. P. 2000, MNRAS, 319, 255

Linnell, A. P., Hubeny, I., \& Harmanec, P. 1998, ApJ, 509, 379

Linnell, A. P., \& Hubeny, I. 1996, ApJ, 471, 958

Mathis, J. S. 1990, ARA\&A, 28, 37

Mazzali, P. A., Pauldrach, A. W. A., Puls, J., \& Plavec, M. J. 1992, A\&A, 254, 241

Reynolds, S. P. 1986, ApJ, 304, 713

Rodriguez, L. F., Ho, P. T. P., Torrelles, J. M., Curiel, S., \& Cantò, J. 1990, ApJ, 352, 645

Seaquist, E. R., \& Taylor, A. R. 1990, ApJ, 349, 313

Schmid-Burgk, J. 1982, A\&A, 108, 169

Stevens, I. R. 1995, MNRAS, 277, 163

Taylor, A. R., \& Seaquist, E. R. 1984, ApJ, 286, 263

Umana, G., Maxted, P. F. L., Trigilio, C., et al. 2000, A\&A, 358, 229

Umana, G., Trigilio, C., \& Leone, F. 2002, in preparation

Viotti, R., Ferrari-Toniolo, M., Marcocci, M., et al. 1978, A\&A, 62, 28

Wade, C. M., \& Hjellming, R. M. 1972, Nature, 235, 270

Williams, P. M. 1996, in Radio Emission from the Stars and the Sun, ed. A. R. Taylor, \& J. M. Paredes, ASP Conf. Ser., 93, 15

Wilson, R. E. 1974, ApJ, 189, 319

Wright, A. E., \& Barlow, M. J. 1975, MNRAS, 170, 41

Zeilik, M., Heckert, P., Henson, G., \& Smith, P. 1982, AJ, 87, 1304 